\title{
¿LOS FONEMAS / v / y / / PRESENTAN ALÓFONOS aproximantes en el Chedungun hablado en el Alto Bío-BÍO? EVIDENCIAS DESDE UNA PERSPECTIVA ACÚSTICA*
}

\author{
Darío Fuentes-Grandón** \\ Gastón Salamanca Gutiérrez***
}

\section{Resumen}

Este trabajo estudia las realizaciones aproximantes de los fonemas /v/ y /ð/ del chedungun hablado en Alto Bío-Bío, una variedad del mapudungun. A través de grabaciones y filmaciones de nueve colaboradores, se realizó una descripción articulatoria y espectrográfica de sus realizaciones. Se utilizaron scripts de Praat durante los procesos de normalización, etiquetaje y análisis acústico. A través de las diferencias de intensidad de las realizaciones con su contexto vocálico, se pudo comprobar, desde una perspectiva acústica, la existencia de realizaciones aproximantes de /v/ y / / en el chedungun de Alto Bío-Bío.

Palabras clave: mapudungun, aproximantes, fonética acústica, scripts.

\section{Do / v / AND / / PHONEMES APPROXIMANT ALLOPHONES in Alto Bío-bíos Chedungun? Evidences from AN ACOUSTIC PERSPECTIVE}

\begin{abstract}
This work studied the approximant realizations of phonemes /v/ and /ð/ of Alto BíoBío's chedungun, a variety of mapudungun. The first articulatory and spectrographic descriptions were made through audio and audiovisual recordings from nine collaborators. Praat scripts were used during the normalization, labeling and acoustic analysis process. The intensity differences between the consonant and its vocalic context proved, from an acoustic perspective, the existence of approximant sounds of / v/ and /ð/ in Alto Bío-Bío's chedungun.
\end{abstract}

Keywords: mapudungun, approximants, acoustic phonetics, scripts.

* $\quad$ Este artículo se inserta en el contexto del Proyecto FONDECYT 1131095.

** Chileno. Magíster en Lingüística Aplicada de la Universidad de Concepción. dario.fuentes. grandon@gmail.com

***Chileno. Doctor en Lingüística de la Universidad de Concepción. gsalaman@udec.cl

Valgan aquí las mismas referencias indicadas para /ð/. 


\section{Introducción}

Sobre el estatus de los fonos aproximantes en el mapudungún (o del debilitamiento de los fonemas, en general), no hay mayores estudios, probablemente debido al uso relativamente reciente de esta etiqueta, lo cual es particularmente cierto si nos referimos a los fonemas descritos sistemáticamente como fricativos $/ \mathrm{v} /(/ \mathrm{f} /)$ y $/ ð /(/ \theta /)$.

En efecto, sobre /ð/, se han reportado realizaciones post o interdentales; ensordecidas o sordas y sonoras; pero siempre fricativas (Echeverría, 1964; Salas, 1976; Lagos, 1981; Salamanca, 1997; Henríquez y Salamanca, 2012; Sadowsky et al, 2013; entre otros estudios prominentes). Para /v/, por su parte, se han descrito realizaciones labiodentales o bilabiales; ensordecidas o sordas, y sonoras; también con un modo de articulación fricativo ${ }^{1}$.

Como se observa, respecto del mapudungún, no se han registrado realizaciones aproximantes para $/ \mathrm{v} / \mathrm{ni}$ para $/ ð /{ }^{2}$. Sin embargo, de la interacción con hablantes nativos de distintos lugares de la Araucanía se puede hipotetizar que tales segmentos ocurren de manera consistente.

El artículo que nos ocupa, precisamente, entrega evidencia acústica que releva la pertinencia de utilizar esta etiqueta en el caso de los fonemas /v/ y /ð/; específicamente, en la zona de habla mapuche pehuenche emplazada en la comuna de Alto Bío-Bío.

\section{Marco de referencia}

El modo de articulación aproximante ha sido motivo de debate y se pueden encontrar diversas definiciones para el mismo. Ladefoged (1964), Piñeros (2009), Sola Prado (2011) y Martínez-Celdrán (2013) proponen diversos conceptos que no calzan, del todo, entre ellos. Entre las definiciones, se destaca la de Martínez-Celdrán, por dar pie a una subdivisión de las aproximantes:

1 Valgan aquí las mismas referencias indicadas para /ð/.

2 Es interesante observar que estudios recientes (Borland Delorne, 2004; Pérez, 2007; Sadowsky y Salamanca, 2011; entre otros) reportan una amplia serie de fonos aproximantes en el español de Chile -entre ellos [v] y [ð]-, variante del español con la que el mapudungún está en contacto. 
Las aproximantes son los segmentos que, poseyendo cierto grado de constricción, carecen de la precisión articulatoria requerida para producir una corriente turbulenta de aire, bien sea por la falta de suficiente tensión en los órganos articuladores, bien sea porque el tracto vocal no está suficientemente constreñido o ambas cosas conjuntamente. (Martínez Celdrán, 2013: 15).

La definición anterior incorpora una característica adicional y poco convencional al término, al considerar como una aproximante no solo aquella cuyos órganos articulatorios no están constreñidos, sino también aquella que, aun cuando estos lo están, no es capaz de generar un ruido de fricción por falta de tensión articulatoria. Por otra parte, se releva la ausencia de la turbulencia de aire que, como señala Sola Prado (2011), permitiría diferenciar las fricativas de las aproximantes, puesto que en estas últimas no se encuentra ruido de fricción, "sino simplemente una disminución de la intensidad de los formantes vocálicos que las rodean" (121), a diferencia de las fricativas, en las que:

se da un estrechamiento del canal bucal a la salida del aire, no lo suficientemente estrecho como para causar una oclusión, pero lo bastante cerrado como para que se produzca una fricción, que en el plano acústico se percibe como un tipo de ruido, y en el espectrograma se representa con un aspecto poco nítido, debido a la turbulencia de las ondas sonoras. (Sola Prado, 2011: 121).

Sin embargo, la mera diferencia entre fricativas y aproximantes no es suficiente para esclarecer y segmentar el continuo de debilitamiento articulatorio que implica. A pesar del carácter poco convencional de la definición de aproximante propuesta por Martínez-Celdrán, a partir de esta se propone una clasificación de aproximantes basada en la distancia de los órganos articulatorios, en el grado de tensión y en algunas características acústicas. Esta diferencia se considera útil desde un punto de vista metodológico, pues permite despejar dudas con respecto a las diversas características acústico-articulatorias que presentan esta clase de sonidos, además de proponer una etiqueta posible para aquellas realizaciones que se encuentran entre la categoría "fricativa" y la tradicional "aproximante", esto es, aquella realización que, juntando los órganos articulatorios, no es capaz de producir ruido de fricción. A continuación se citan las definiciones de dichas clases: 
a) Aproximantes abiertas: "aquellas que poseen una estructura de formantes transicionales entre las vocales adyacentes y que, como tales, se manifiestan con pulsos glotales débiles y que poseen una breve duración si se compara con las vocales vecinas y una menor intensidad" (Martínez Celdrán, 2013: 17).

b) Aproximante cerrada: "Se caracteriza por tener unos pulsos glotales, por encima de la barra de sonoridad, muy débiles que llegan a desaparecer en muchos casos. Se diferencia de la oclusiva porque no posee explosión. Desde un punto de vista articulatorio los órganos pueden estar cerrados pero sin ejercer presión entre ellos, lo cual impide la explosión, incluso a veces ese cierre es muy breve y quedan restos de pulsos en ambos lados"' (Martínez Celdrán, 2013: 18).

c) Aproximante vocálica: respecto al espectrograma que usa como ejemplo, el autor señala que "posee todo él mayor intensidad y se marcan menoslas a proximantes al tener menor diferencia deintensidad con las vocales vecinas; (...) Ciertamente es difícil segmentar dicho sonido pues las fronteras con las vocales vecinas son muy borrosas. La aproximante se percibe perfectamente por vía auditiva, a pesar de su imagen poco definida. Solo la curva de intensidad superpuesta nos permite adivinar dónde se sitúa" (Martínez Celdrán, 2013: 19).

Hasta ahora, una característica común con respecto al comportamiento de las aproximantes pareciera ser el relajamiento articulatorio, incluso considerando las aproximantes cerradas, cuyos órganos articulatorios tendrían menos tensión que las fricativas. Un índice acústico que se relaciona de manera inversamente proporcional a la constricción articulatoria es la intensidad. Pareciera entonces que tanto la disminución como el aumento de la intensidad en relación con la vocal que antecede y sucede a la aproximante, respectivamente, sería un índice que permitiría caracterizar a las aproximantes, como proponen Martínez Celdrán (1998: 71; 2013: 21), Sola Prado (2011: 38), Figueroa Candia (2012: 42) y Simonet et al (2012). 


\section{Metodología}

\subsection{Toma de muestra}

a) Corpus de habla: en la presente investigación, se trabajó con un corpus de habla del Proyecto Fondecyt $\mathrm{N}^{\circ} 1131095$. Esta muestra se compone del registro sonoro y audiovisual de más de 100 ítems léxicos de 30 hablantes nativos de chedungun, 15 del sector del Valle del Queuco y 15 del sector del Valle del río Bío-Bío.

b) Recolección de datos: la recolección de datos se realizó en 10 localidades de Alto Bío-Bío. Se trabajó con las comunidades de Pitril, Cauñicu, Malla Malla, Trapa Trapa y Butalelbún (por el sector del río Queuco); y Avellano, Chenqueco, Quiñelón, Chevquelavken, y Huallalí-El Barco (por el sector del río Bío-Bío). Es importante destacar que la inclusión de zonas del curso del río Bío-Bío es un aporte relevante a la literatura sobre el chedungun, ya que en la actualidad no hay reportes sobre la fonología de la variante hablada en esa zona ${ }^{3}$.

En cuanto a los materiales para la recolección de datos, se utilizó una cámara Handycam Sony DVD108/DVD308/DVD608/DCD708, además de una grabadora TASCAM DR-100. Es decir, se realizó tanto un registro acústico como uno audiovisual.

c) Colaboradores: los colaboradores fueron seleccionados de acuerdo con su competencia en la lengua vernácula, así como también por no tener ningún tipo de impedimento físico o mental para las tareas solicitadas.

d) Instrumentos: el instrumento utilizado en esta investigación fue una lista léxica que tiene como base la aplicada por Croese (1980), para postular la división dialectal, además de incluir ítems que contienen los segmentos problemáticos en las descripciones de la zona.

\subsection{Normalización de señales}

La intensidad de la señal y ruidos de altas y bajas frecuencias se modificaron utilizando el script creado por Chad Vicenik, que aplica un

3 Es por ello, entre otros factores, que para este trabajo se seleccionaron 9 colaboradores provenientes de este subsector. 
low-pass filter y regula la intensidad de los audios en Praat (Boersma y Weenink, 1992), para que además de ello hicera un high-pass filter. De este modo, se reguló la intensidad de todos los audios alrededor de los $70 \mathrm{~dB}$, se borraron los sonidos por sobre los $15000 \mathrm{~Hz}$ y por bajo los 60 $\mathrm{Hz}$. Las diferencias en los máximos de $\mathrm{Hz}$ considerados durante cada análisis ( $5000 \mathrm{~Hz}$ para hombres, $5500 \mathrm{~Hz}$ para mujeres) fueron regulados al momento mismo de hacer el análisis a través del script elaborado por Figueroa Candia (2012).

\subsection{Segmentación y etiquetaje de la señal}

Dado que se utilizó el script elaborado por Figueroa Candia (2012) para el análisis de las aproximantes, fue necesario que las etiquetas estuvieran en función de los procedimientos de dicho script. Se utilizó una versión modificada del script de Katherine Crosswhite, que ya había sido modificado por Mark Antoniou, el cual genera automáticamente archivos textgrids para todos los archivos de audios en una misma carpeta. La modificación que se hizo de este script consistió en hacer que los archivos textgrids resultantes presentaran las características requeridas por el script de Figueroa Candia. Así, todos los archivos textgrid utilizados tuvieron el mismo número de tiers con los distintos nombres y del mismo tipo (todos tiers de intervalos, excepto el tier número cuatro que fue de puntos), esto es, un total de cinco tiers.

Se siguió una metodología exhaustiva para el etiquetaje de las realizaciones ${ }^{4}$, lo que implicó el etiquetaje de su contexto y de las transiciones, puesto que el script extraería, de estos tiers, información relevante para el análisis acústico. Para el etiquetaje de los límites de la aproximante y del contexto fonético, se tomó como referencia la forma de onda del oscilograma (Figueroa Candia, 2012: 41-42); se observó cuidadosamente la forma de onda en el centro de la aproximante y de los sonidos del contexto fonético, luego se marcó el límite en el último ciclo de la forma de onda que mantenía una estructura similar al comportamiento de los ciclos de la aproximante o de los sonidos del contexto fonético.

4 Se debe mencionar que también fueron etiquetados sonidos que no son aproximantes, así como contextos distintos del intervocálico, aun cuando Figueroa Candia plantea su script para el análisis de aproximantes en contexto intervocálico. Esto se hizo para poder abarcar de manera más completa las realizaciones de los fonemas estudiados. 
En el caso de las transiciones, se marcó, en el sonido previo a la aproximante, el punto en el que los formantes cambian su trayectoria; luego, en la aproximante, se etiquetó el punto en el que los formantes se estabilizan; así, este primer intervalo conforma la primera transición; la segunda transcición, que también corresponde a un intervalo, fue etiquetada utilizando el mismo procedimiento, pero de forma inversa; esto es, dentro de la aproximante, se etiquetó el punto en el que los formantes se desestabilizaban e iniciaban su trayectoria hacia los formantes del sonido siguiente; luego se etiquetó el punto en el que los formantes se estabilizaban dentro del sonido siguiente.

Para describir de la manera más fidedigna posible las realizaciones de los fonemas a estudiar, se recurrió a un análisis auditivo, espectrográfico y visual. En primer lugar, se escuchó la grabación y examinó el espectrograma en Praat, para poder determinar las características acústicas de cada realización. Para el etiquetaje de las aproximantes, se realizó un análisis espectrográfico en base a las características propuestas por Martínez Celdrán para las tres clases de aproximantes: cerradas, abiertas y vocálicas. Luego, se examinó detalladamente cada filmación, con el propósito de corroborar y corregir el etiquetaje anteriormente realizado; en especial, aquella información relativa a la zona de articulación. Luego de esta etapa, se recolectaron los índices acústicos utilizando el script para la medición de aproximantes elaborado por Figueroa Candia (2012).

\subsection{Selección de índices acústicos}

El script de Figueroa Candia arroja una amplia cantidad de índices acústicos, pero no se utilizaron todos durante la investigación. En relación con lo revisado en la teoría y con las catacterísticas de esta investigación, se seleccionaron los valores de la intensidad máxima de los sonidos que rodean a la realización y el punto de intensidad mínima de la realización. Estos fueron escogidos porque la literatura señala que las diferencias de intensidad entre el contexto vocálico y la realización guardan una relación con el grado de constricción de la consonante. Por otra parte, al seleccionar y medir los puntos máximos y mínimos de intensidad, ni se supone la existencia de fronteras de sonidos ni estos puntos se acercan a estas zonas difusas, que pudieran ser de un sonido $u$ otro, por lo que se estaría trabajando sobre la base de un índice que indiscutiblemente 
pertenece a la realización en cuestión.

Es necesario aclarar que, para medir las diferencias de intensidad, fue necesario realizar dos procesos. Uno fue la reducción de la muestra a todas aquellas realizaciones que presentaran contexto intervocálico, dado que eso permitiría rodear la realización en cuestión por un grupo de vocales que presentan un grado de constricción nulo; esto haría que todas las diferencias de intensidad se midieran con el mismo punto de referencia (constricción nula). Otro fue calcular un índice relativo: a través de una regla de tres simple, teniendo en cuenta los puntos de intensidad, se calcularon el porcentaje de disminución de intensidad desde el punto máximo de la vocal anterior al punto mínimo de la realización y el porcentaje de aumento de intensidad desde el punto mínimo de la realización al máximo de la vocal siguiente. Así se obtuvo dos índices relativos relacionados al grado de constricción que permitirían la relación y comparación entre distintos tipos de realizaciones.

\section{Resultados}

\subsection{Realizaciones aproximantes según fonemas.}

Los gráficos 1 y 2 resumen el recuento de las realizaciones aproximantes de / v / y / /. Este fue el resultado del análisis espectrográfico, auditivo y visual, que permitió clasificar las aproximantes según las distintas clases (cerradas, abiertas y vocálicas) y según su zona de articulación.

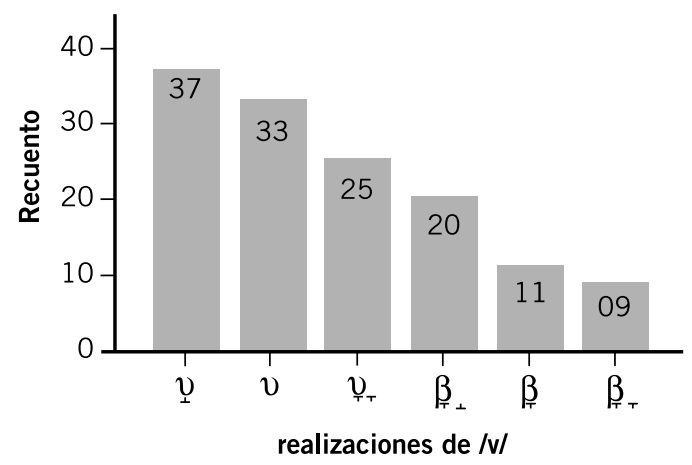

Gráfico 1 - Aproximantes de / v / 


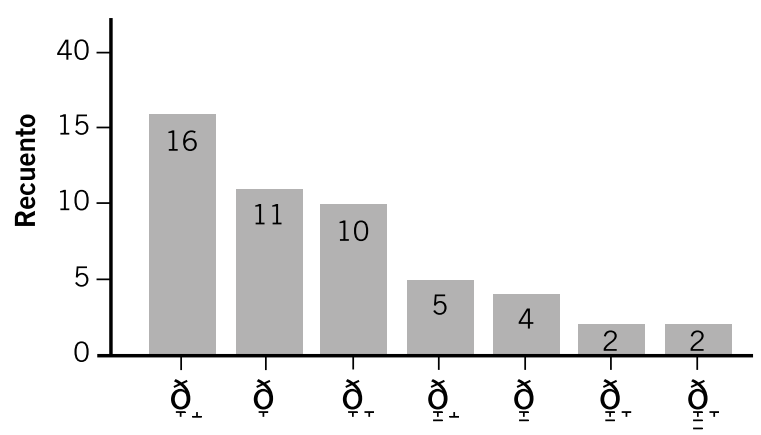

realizaciones de /ð/

Gráfico 2 - Aproximantes de /ð/

El gráfico 1 da cuenta de las aproximantes labiodentales y bilabiales de $/ \mathrm{v} /$, que corresponden al $45,82 \%$ de las realizaciones registradas para este fonema. Las aproximantes con mayor recuento corresponden al grupo de las labiodentales, encabezado por la cerrada [v]], seguida por la abierta $[v]$ y finalmente, con un menor recuento, se encuentra la vocálica [ṿ* ]. El grupo con un menor recuento corresponde al de las aproximantes bilabiales, encabezado por la cerrada $\left[\beta^{*}\right]$, la abierta $[\beta]$ y la vocálica [ßß"].

El gráfico 2 da cuenta de las aproximantes interdentales, postdentales y alveolares de /ð/, que corresponden al 29,41\% de sus realizaciones registradas. El grupo con mayor tamaño corresponde al interdental, cuya aproximante con mayor recuento fue la cerrada [ $\left.\partial_{\tau}^{\mathrm{I}}\right]$, seguida por la abierta [ð̋] y seguida, a su vez, por la vocálica [ợ- ]. El segundo grupo con mayor recuento es el de las postdentales, que es encabezado por la cerrada [ợ , seguida por la abierta [ợ] y, en último lugar se ubica la

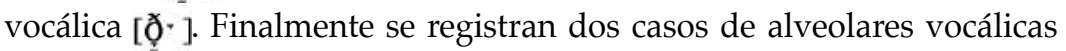
[ర్ָ๋ ].

\subsection{Valores de intensidad de las aproximantes en contexto vocálico}

A continuación, se presentan los gráficos que resumen los resultados de los índices acústicos relacionados con la intensidad de las distintas aproximantes registradas con contexto vocálico. El gráfico 3 compara los 
puntos de intensidad máximos de las vocales que anteceden y suceden a la aproximante con el punto de intensidad mínimo de la aproximante. Es importante recordar que, a partir de ahora, se utilizarán los resultados filtrados.

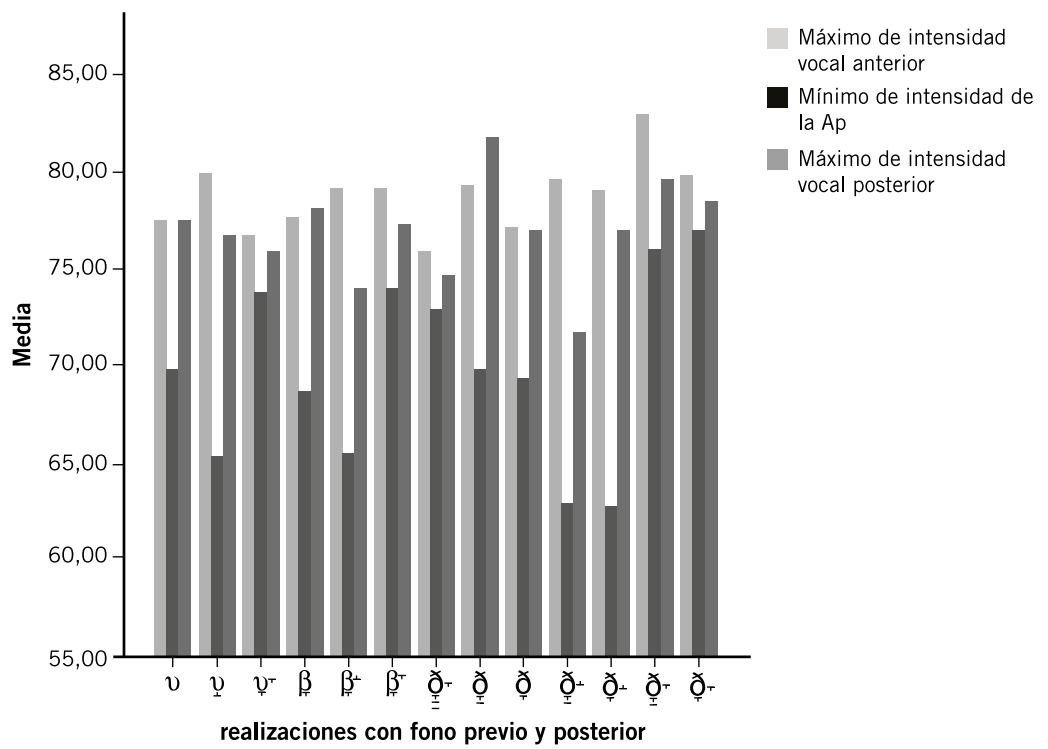

Gráfico 3 - Medias de los puntos máximos y mínimos de intensidad de la aproximante y su contexto vocálico

En el gráfico 3, se observa que el punto de mínima intensidad de la aproximante es, en todos los casos, inferior a la intensidad registrada en su contexto vocálico. Esto era de esperarse debido a que la intensidad disminuye en la medida que aumenta la constricción articulatoria; es decir, es normal que una aproximante, cuyos órganos articulatorios se encuentran más cerca unos de otros, presente menor intensidad que las vocales, cuyos órganos articulatorios se encuentran más separados.

Otra tendencia a destacar son las diferencias de intensidad entre el contexto y la aproximante, dependiendo de la clase de aproximante. Las aproximantes cerradas son las que presentan un menor grado de intensidad y una mayor diferencia entre el punto mínimo de intensidad con los valores de intensidad del contexto vocálico. Las aproximantes vocálicas presentan las diferencias más bajas entre su punto mínimo de intensidad y el contexto vocálico, y, por lo mismo, los niveles de intensidad más altos. Finalmente, las aproximantes abiertas se encuentran 
siempre entre las tendencias mostradas por los valores de intensidad de aproximantes cerradas y vocálicas.

A continuación, el gráfico 4 da cuenta de los índices relativos de intensidad. Cabe mencionar que estos índices permiten relacionar el contexto con la aproximante $y$, al ser índices relativos, permiten comparar los porcentajes de aumento y disiminución de intensidad de distintas aproximantes.

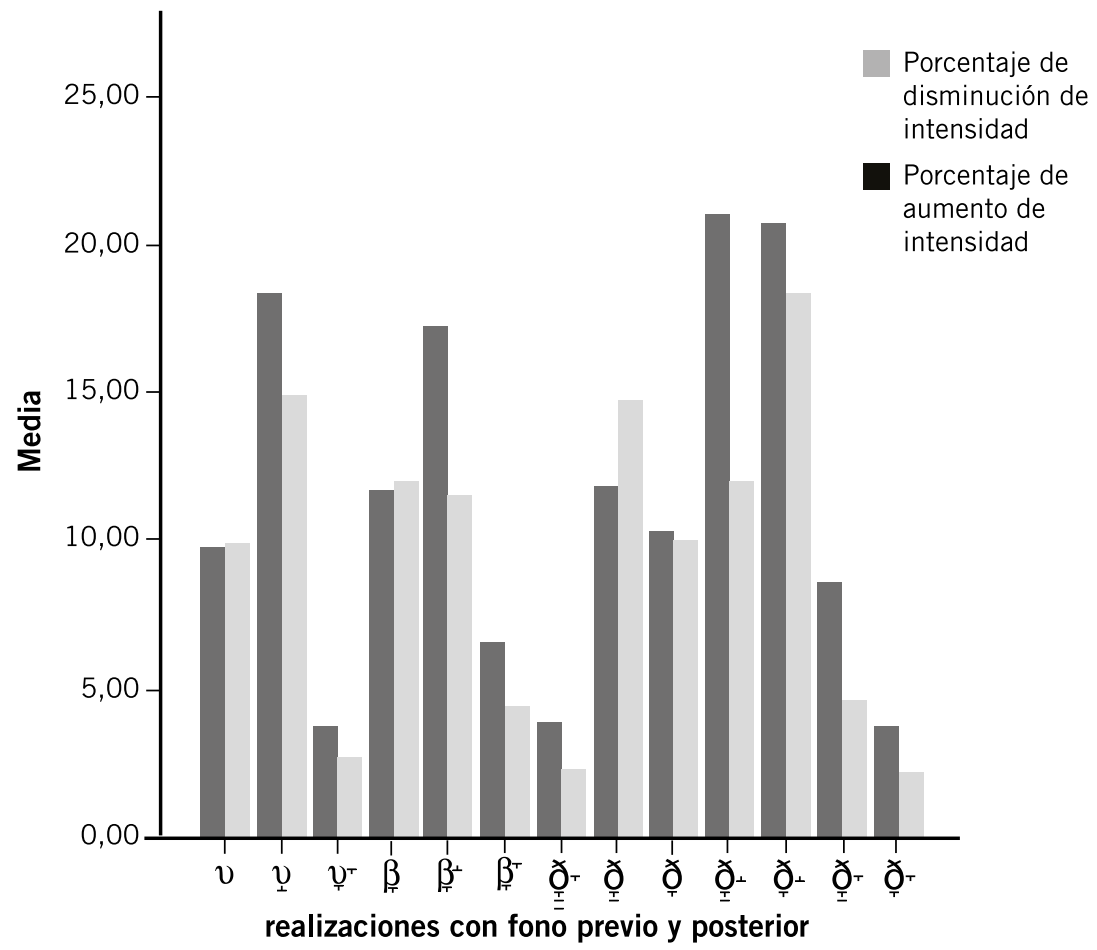

Gráfico 4 - Medias de los índices relativos de disminución y aumento de intensidad

Pareciera haber cierta similitud en los porcentajes según los distintos tipos de aproximantes. En general, las aproximantes cerradas se acercan al 15\% de disminución y aumento, las aproximantes abiertas rodean el $10 \%$ y las aproximantes vocálicas casi no superan el 5\%. Para graficar mejor la tendencia anteriormente mencionada, y considerando que los valores se encuentran en índices relativos, se han juntado todas las 
realizaciones aproximantes de /v/ y /ð/ con contexto vocálico completo y se han dividido en las tres clases de aproximantes. Los resultados se encuentran en el gráfico 5 .

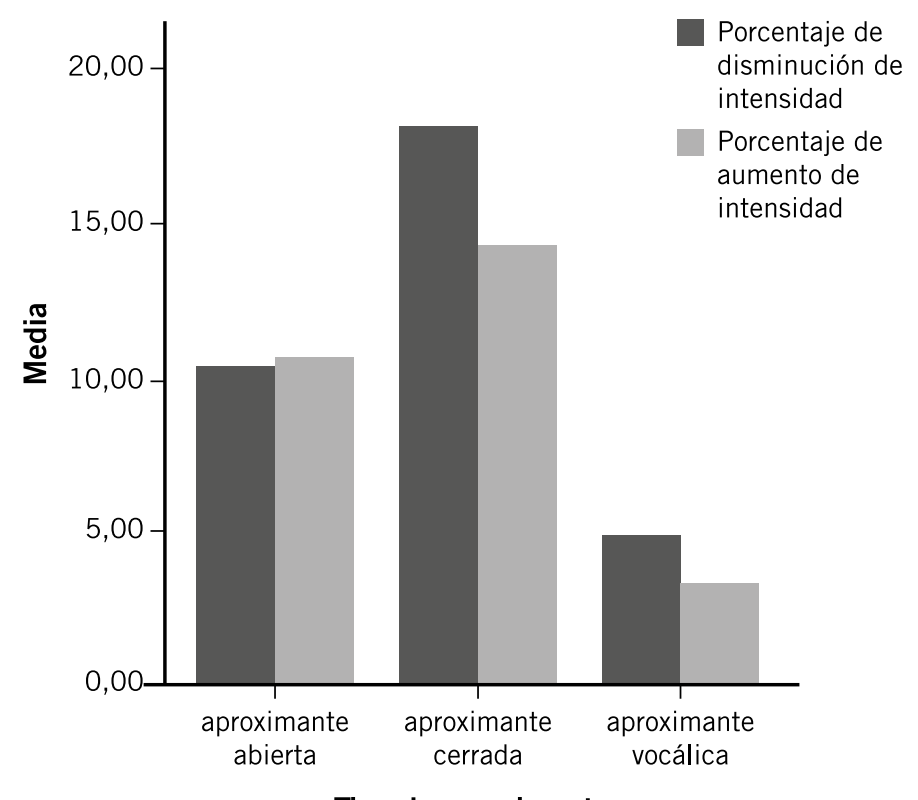

Tipo de aproximante

Gráfico 5 - Medias de los índices relativos de disminución y aumento de intensidad en los tipos de aproximantes

\subsection{Análisis de las diferencias de intensidad entre aproximantes con contexto vocálico completo}

A partir de estos dos índices relativos, se han realizado dos análisis de sus varianzas (ANOVA), considerando como factores los tres distintos tipos de aproximantes registrados en las realizaciones con contexto vocálico. Las ANOVAS señalaron que las diferencias son significativas; específicamente, estos resultados pueden ser vistos en la Tabla 1. Se utilizaron análisis post-hoc para conocer las diferencias específicas, los cuales fueron, según lo indicado por el estadístico de Levene (ver Tabla 2), la prueba de Tukey para la disminución de intensidad (variable en la que sí se puede suponer igualdad de varianzas) y la prueba de Games- 
Howell para el aumento de la intensidad (variable en la que no se puede suponer igualdad de varianzas). La Tabla 3 da cuenta de los resultados del análisis post-hoc, y los gráficos 6 y 7 ofrecen una mejor visualización de las diferencias de las medias de las clases de aproximantes para cada uno de los distintos índices relativos. En la Tabla 4, la prueba de Tukey estableció subconjuntos de las clases de aproximantes según el aumento de la intensidad y las ordenó siguiendo el continuo de constricción.

\begin{tabular}{|c|c|c|c|c|c|c|}
\hline Variable & & $\begin{array}{l}\text { Suma de } \\
\text { cuadrado }\end{array}$ & $\mathrm{gl}$ & $\begin{array}{c}\text { Media } \\
\text { cuadrática }\end{array}$ & F & Sig. \\
\hline \multirow{3}{*}{$\begin{array}{l}\text { Porcentaje de } \\
\text { disminución de } \\
\text { intensidad }\end{array}$} & Entre grupos & 3737,651 & 2 & 1868,825 & 67,268 & ,000 \\
\hline & $\begin{array}{l}\text { Dentro de } \\
\text { grupos }\end{array}$ & 3444,938 & 124 & 27,782 & & \\
\hline & Total & 7182,589 & 126 & & & \\
\hline \multirow{3}{*}{$\begin{array}{l}\text { Porcentaje de } \\
\text { aumento de } \\
\text { intensidad }\end{array}$} & Entre grupos & 2417,281 & 2 & 1208,641 & 45,875 & ,000 \\
\hline & $\begin{array}{l}\text { Dentro de } \\
\text { grupos }\end{array}$ & 3266,945 & 124 & 26,346 & & \\
\hline & Total & 5684,227 & 126 & & & \\
\hline
\end{tabular}

Tabla 1 - ANOVA de las diferencias de los índices relativos de descenso y aumento de intensidad según las distintas clases de aproximantes

\begin{tabular}{lcccc}
\hline Variable & $\begin{array}{l}\text { Estadístico } \\
\text { de Levene }\end{array}$ & df1 & df2 & Sig. \\
\hline $\begin{array}{l}\text { Porcentaje de disminución de } \\
\text { intensidad }\end{array}$ & 3,024 & 2 & 124 &, 052 \\
\hline $\begin{array}{l}\text { Porcentaje de aumento de } \\
\text { intensidad }\end{array}$ & 3,548 & 2 & 124 &, 032 \\
\hline
\end{tabular}

Tabla 2 - Prueba de homogeneidad de varianzas de los índices relativos de descenso y aumento de intensidad según las distintas clases de aproximantes 


\begin{tabular}{|c|c|c|c|c|c|c|c|}
\hline \multirow{2}{*}{$\begin{array}{l}\text { Variable } \\
\text { dependiente }\end{array}$} & \multirow{2}{*}{$\begin{array}{l}\text { Variante de } \\
\text { referencia (I) }\end{array}$} & \multirow{2}{*}{$\begin{array}{c}\text { Variante } \\
\text { comparada } \\
\text { (j) }\end{array}$} & \multirow{2}{*}{$\begin{array}{c}\text { Diferencia } \\
\text { de medias } \\
(\mathrm{I}-\mathrm{J})\end{array}$} & \multirow{2}{*}{$\begin{array}{l}\text { Error } \\
\text { estándar }\end{array}$} & \multirow{2}{*}{ Sig. } & \multicolumn{2}{|c|}{$\begin{array}{l}95 \% \text { de intervalo } \\
\text { de confianza }\end{array}$} \\
\hline & & & & & & $\begin{array}{l}\text { Límite } \\
\text { inferior } \\
\end{array}$ & $\begin{array}{c}\text { Límite } \\
\text { superior } \\
\end{array}$ \\
\hline \multirow{6}{*}{$\begin{array}{l}\text { Porcentaje de } \\
\text { disminución } \\
\text { de intensidad } \\
\text { (Tukey) }\end{array}$} & \multirow{2}{*}{$\begin{array}{l}\text { aproximante } \\
\text { abierta }\end{array}$} & $\begin{array}{l}\text { aproximante } \\
\text { cerrada }\end{array}$ & $7,84299^{*}$ & 1,08951 & , 000 & $-10,4275$ & $-5,2585$ \\
\hline & & $\begin{array}{l}\text { aproximante } \\
\text { vocálica }\end{array}$ & $5,52784^{*}$ & 1,21379 & ,000 & 2,6485 & 8,4072 \\
\hline & \multirow{2}{*}{$\begin{array}{l}\text { aproximante } \\
\text { cerrada }\end{array}$} & $\begin{array}{c}\text { aproximante } \\
\text { abierta }\end{array}$ & $7,84299^{*}$ & 1,08951 & ,000 & 5,2585 & 10,4275 \\
\hline & & $\begin{array}{l}\text { aproximante } \\
\text { vocálica }\end{array}$ & $13,37083^{*}$ & 1,18216 & ,000 & 10,5665 & 16,1751 \\
\hline & \multirow{2}{*}{$\begin{array}{l}\text { aproximante } \\
\text { vocálica }\end{array}$} & $\begin{array}{l}\text { aproximante } \\
\text { abierta }\end{array}$ & $-5,52784^{*}$ & 1,21379 & ,000 & $-8,4072$ & $-2,6485$ \\
\hline & & $\begin{array}{c}\text { aproximante } \\
\text { cerrada }\end{array}$ & $-13,37083^{*}$ & 1,18216 & ,000 & $-16,1751$ & $-10,5665$ \\
\hline \multirow{6}{*}{$\begin{array}{l}\text { Porcentaje de } \\
\text { aumento de } \\
\text { intensidad } \\
\text { (Games- } \\
\text { Howell) }\end{array}$} & \multirow{2}{*}{$\begin{array}{l}\text { aproximante } \\
\text { abierta }\end{array}$} & $\begin{array}{c}\text { aproximante } \\
\text { cerrada }\end{array}$ & $-3,65346^{*}$ & 1,12025 & ,004 & $-6,3244$ &,- 9826 \\
\hline & & $\begin{array}{c}\text { aproximante } \\
\text { vocálica }\end{array}$ & $7,33982^{*}$ & ,92902 & ,000 & 5,1178 & 9,5618 \\
\hline & \multirow{2}{*}{$\begin{array}{l}\text { aproximante } \\
\text { cerrada }\end{array}$} & $\begin{array}{c}\text { aproximante } \\
\text { abierta }\end{array}$ & $3,65346^{*}$ & 1,12025 & ,004 & ,9826 & 6,3244 \\
\hline & & $\begin{array}{l}\text { aproximante } \\
\text { vocálica }\end{array}$ & $10,99328^{*}$ & 1,10768 & ,000 & 8,3480 & 13,6385 \\
\hline & \multirow{2}{*}{$\begin{array}{l}\text { aproximante } \\
\text { vocálica }\end{array}$} & $\begin{array}{c}\text { aproximante } \\
\text { abierta }\end{array}$ & $-7,33982^{*}$ & 92902 & ,000 & $-9,5618$ & $-5,1178$ \\
\hline & & $\begin{array}{l}\text { aproximante } \\
\text { cerrada }\end{array}$ & $-10,99328^{*}$ & 1,10768 & ,000 & $-13,6385$ & $-8,3480$ \\
\hline
\end{tabular}

Tabla 3 - Pruebas post-hoc de los índices relativos de descenso y aumento de la intensidad según distintos tipos de aproximantes

\begin{tabular}{lllllr}
\hline Post-hoc & Tipo de aproximante & $\mathrm{N}$ & \multicolumn{3}{c}{ Subconjunto para alfa $=0.05$} \\
\cline { 3 - 6 } & & & 1 & 2 & 3 \\
\hline HSD \\
Tukeya,b & aproximante vocálica & 33 & 3,3140 & & \\
\cline { 2 - 6 } & aproximante abierta & 44 & & 10,6538 & \\
\cline { 2 - 6 } & aproximante cerrada & 50 & & & 1,3072 \\
\cline { 2 - 6 } & Sig. & & 1,000 & 1,000 & 1,000 \\
\hline
\end{tabular}

a. Utiliza el tamaño de la muestra de la media armónica $=41,079$.

b. Los tamaños de grupo no son iguales. Se utiliza la media armónica de los tamaños de grupo. Los niveles de error de tipo I no están garantizados.

Tabla 4 - Subconjuntos según el índice relativo del aumento de intensidad 


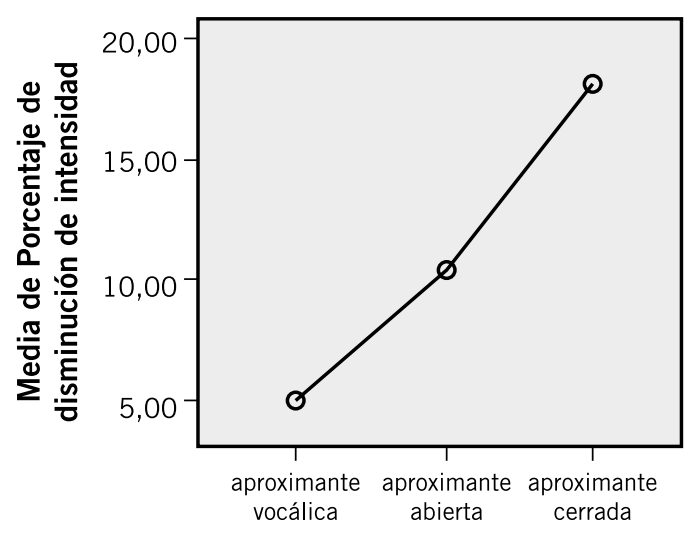

Tipo de aproximante

Gráfico 6 - Diferencias de medias de los índices relativos de la disminución de la intensidad

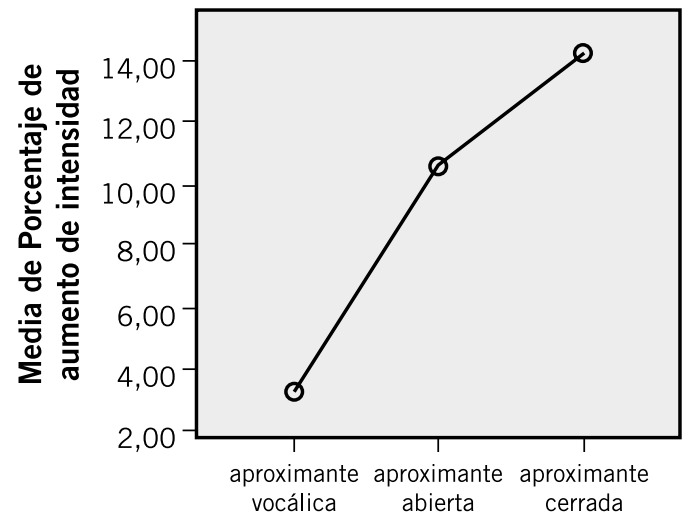

Tipo de aproximante

Gráfico 7 - Diferencias de medias de los índices relativos del aumento de la intensidad

Como se puede observar en los resultados, las diferencias no sólo son significativas en las ANOVAS, sino que, según lo que muestran las pruebas post-hoc, lo son también según cada tipo de aproximante. Dicho en otras palabras, los dos índices relativos logran diferenciar entre sí a los distintos tipos de aproximantes, mostrando diferencias significativas de 0,000 o cercanas a este valor. 
Un punto que los argumentos presentados hasta ahora no aclaran es si las realizaciones etiquetadas como aproximantes cerradas corresponden realmente a aproximantes o pudieran pertenecer a la categoría de las fricativas sonoras, que serían más tensas que las aproximantes. Frente a esto, una primera respuesta que intenta aclarar este punto es que las aproximantes cerradas no presentan ruido de fricción, mientras que las fricativas sonoras sí, según los registros encontrados. A continuación, las figuras 1 y 2 ilustran la diferencia entre una aproximante cerrada y una fricativa sonora de / $/$ encontradas en estos datos:

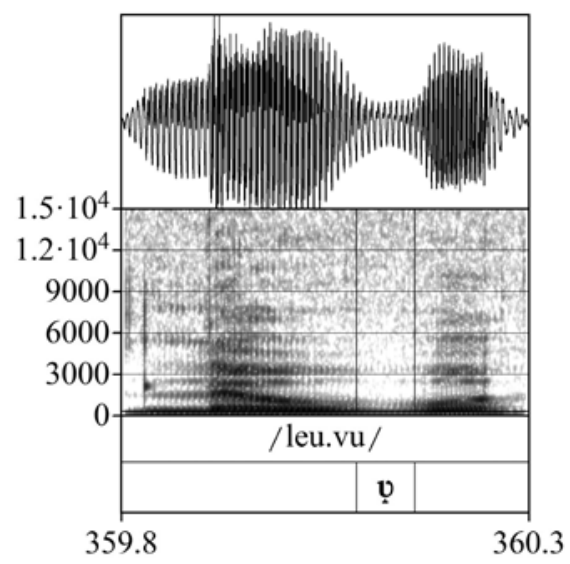

Figura 1 - Espectrograma de [v]

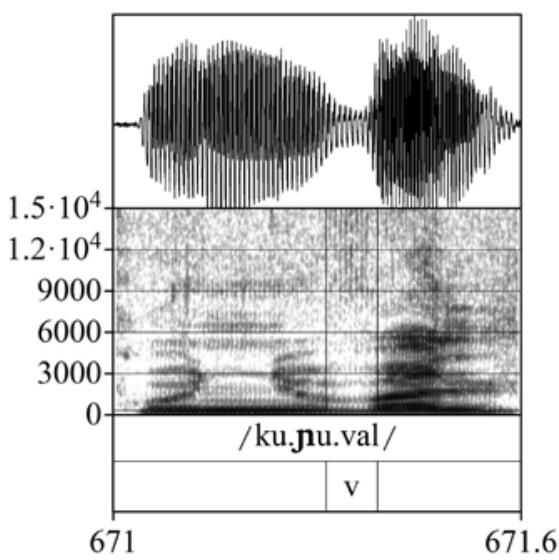

Figura 2 - Espectrograma de [v] 
En ambos espectrogramas se pueden observar las diferencias de cada realización. Así, mientras la aproximante cerrada [v] de la figura 1 tiene unas transiciones bajas y sus formantes comienzan a desaparecer, la frivativa [v] presenta transiciones, pero además, y por sobre ella, alrededor de los $9000 \mathrm{~Hz}$, se encuentra lo que sería el ruido de fricción de esta realización ${ }^{5}$.

Aun cuando los argumentos y la evidencia recién presentados parecen tener solidez teórica, se ha decidido poner a prueba la diferencia entre las fricativas sonoras y las aproximantes. Para ello se ha sometido a una prueba de ANOVA a todas las aproximantes y fricativas sonoras de / v / y / / que se encuentran en posición intervocálica, según los valores que presenta el índice relativo del aumento de la intensidad.

Los resultados de esta última prueba arrojaron que sí hay diferencias significativas entre los grupos, $\mathrm{F}(3,227)=72,926, \mathrm{p}=0,000<0,05$. La prueba de Levene indicó que no se puede asumir varianzas iguales $(5,164$, $\mathrm{p}=0,002<0,05)$, por lo que los datos fueron sometidos a una prueba post-hoc de Games-Howell para observar las diferencias específicas entre grupos. La Tabla 5 muestra los resultados de esta prueba.

\begin{tabular}{|c|c|c|c|c|c|c|}
\hline \multicolumn{2}{|c|}{ Tipo de aproximante } & \multirow{2}{*}{$\begin{array}{l}\text { Diferencia } \\
\text { de medias } \\
\quad(\mathrm{I}-\mathrm{J})\end{array}$} & \multirow[t]{2}{*}{$\begin{array}{l}\text { Error } \\
\text { estándar }\end{array}$} & \multirow[t]{2}{*}{ Sig. } & \multicolumn{2}{|c|}{$\begin{array}{l}95 \% \text { de intervalo de } \\
\text { confianza }\end{array}$} \\
\hline $\begin{array}{l}\text { Tipo de } \\
\text { aproximante de } \\
\text { referencia (I) }\end{array}$ & $\begin{array}{c}\text { Tipo de } \\
\text { aproximante } \\
\text { comparada (J) }\end{array}$ & & & & $\begin{array}{l}\text { Límite } \\
\text { inferior }\end{array}$ & $\begin{array}{l}\text { Límite } \\
\text { superior }\end{array}$ \\
\hline \multirow[t]{3}{*}{$\begin{array}{l}\text { aproximante } \\
\text { abierta }\end{array}$} & $\begin{array}{l}\text { aproximante } \\
\text { cerrada }\end{array}$ & $-3,65346^{*}$ & 1,12025 & ,008 & $-6,5875$ &,- 7195 \\
\hline & $\begin{array}{l}\text { aproximante } \\
\text { vocálica }\end{array}$ & $7,33982^{*}$ & ,92902 & ,000 & 4,8980 & 9,7816 \\
\hline & fricativa & $-9,30838^{*}$ & ,95085 & ,000 & $-11,7849$ & $-6,8318$ \\
\hline \multirow[t]{3}{*}{$\begin{array}{l}\text { aproximante } \\
\text { cerrada }\end{array}$} & $\begin{array}{l}\text { aproximante } \\
\text { abierta }\end{array}$ & $3,65346^{*}$ & 1,12025 & ,008 & 7195 & 6,5875 \\
\hline & $\begin{array}{l}\text { aproximante } \\
\text { vocálica }\end{array}$ & $10,99328^{*}$ & 1,10768 & ,000 & 8,0869 & 13,8997 \\
\hline & fricativa & $-5,65492^{*}$ & 1,12606 & ,000 & $-8,5950$ & $-2,7149$ \\
\hline
\end{tabular}

5 Si bien es cierto, este ruido de fricción no es del todo equivalente al ruido de fricción presentado por una fricativa áfona, no es del todo plausible esperar que estos ruidos se parezcan, pues hay que tener en cuenta que en la amalgama de inarmonicidad de la fricativa áfona no puede aparecer en un sonido que tiene una base armónica, como lo es la vibración de las cuerdas vocales. 


\begin{tabular}{llrrrrr}
\hline $\begin{array}{l}\text { aproximante } \\
\text { vocálica }\end{array}$ & $\begin{array}{l}\text { aproximante } \\
\text { abierta }\end{array}$ & $-7,33982^{*}$ &, 92902 &, 000 & $-9,7816$ & $-4,8980$ \\
& $\begin{array}{l}\text { aproximante } \\
\text { cerrada }\end{array}$ & $-10,99328^{*}$ & 1,10768 &, 000 & $-13,8997$ & $-8,0869$ \\
& fricativa & $-16,64820^{*}$ &, 93601 &, 000 & $-19,0928$ & $-14,2036$ \\
\hline fricativa & $\begin{array}{l}\text { aproximante } \\
\text { abierta }\end{array}$ & $9,30838^{*}$ &, 95085 &, 000 & 6,8318 & 11,7849 \\
& $\begin{array}{l}\text { aproximante } \\
\text { cerrada } \\
\text { aproximante } \\
\text { vocálica }\end{array}$ & $5,65492^{*}$ & 1,12606 &, 000 & 2,7149 & 8,5950 \\
& $16,64820^{*}$ &, 93601 &, 000 & 14,2036 & 19,0928 \\
& & & & & & \\
\end{tabular}

*. La diferencia de medias es significativa en el nivel 0.05 .

Tabla 5 - Análisis de las diferencias de medias entre realizaciones fricativas sonoras y aproximantes

En la Tabla 5, se puede comprobar que todos los factores, es decir, los distintos modos de articulación involucrados, se diferenciaron de los otros modos de manera significativa. Esto prueba que las fricativas sonoras, como categoría que presenta ruido de fricción en el espectrograma, se diferencian en cuanto a tensión articulatoria de las aproximantes cerradas. Con ello, podemos afirmar que las aproximantes cerradas merecen ser etiquetadas como tales, en atención al continuo de las clases de sonidos, a su constricción articulatoria y a las etiquetas disponibles en la literatura.

El gráfico 8 muestra las diferencias de medias de estas clases según el índice relativo de aumento de intensidad en un orden ascendente; arrojando los valores de menos del $5 \%$ para las aproximantes vocálicas, por sobre el 10\% para las aproximantes abiertas, 15\% para las aproximantes cerradas, y $20 \%$ para las fricativas. 


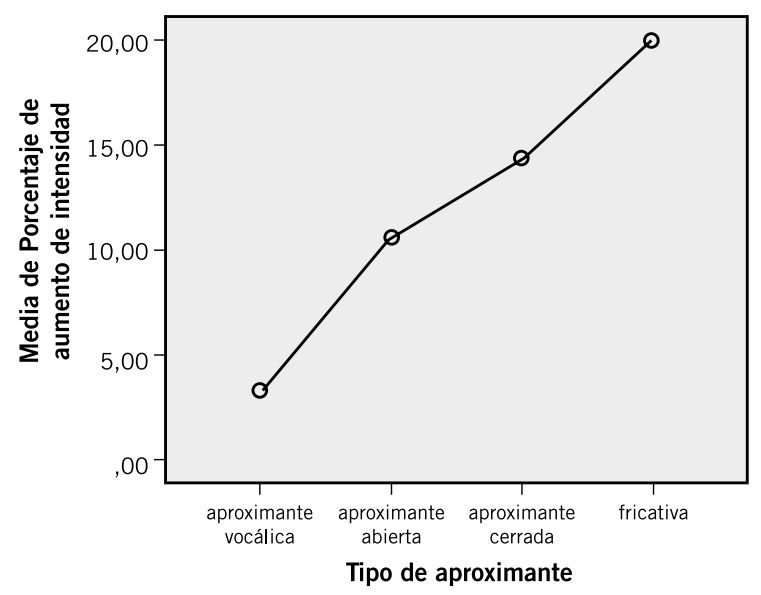

Gráfico 8 - Diferencia de medias del índice relativo del aumento de la intensidad entre fricativas sonoras y aproximantes

\section{Conclusiones}

Como se pudo comprobar, los fonemas /v/ y /ð/ del chedungun hablado en Alto Bío-Bío sí presentan realizaciones aproximantes de las tres clases. Además de ello, tras la prueba de ANOVA realizada entre las realizaciones fricativas sonoras y aproximantes según su índice relativo del aumento de intensidad, la duda con respecto a si presentan realizaciones aproximantes debiera quedar despejada.

En base a lo anterior, es necesario incluir las realizaciones aproximantes como alófonos de /v/ y /ð/ dentro de la variedad registrada. Esto debido al porcentaje no menor que ocupan entre las realizaciones de cada fonema (45,82\% en el caso de /v/ y 29,41\% en el caso de /ð/). También debido a que presentan realizaciones aproximantes cerradas, abiertas y vocálicas; es decir, que todo el continuo de aproximantes se encuentra ya instalado como parte de estas realizaciones.

Finalmente, se puede establecer que la intensidad es un índice acústico capaz de diferenciar de manera significativa a las clases de aproximantes y otros tipos de realizaciones (vocales y fricativas). Se establece un orden, como lo muestran los gráficos, que sigue un continuo del aumento de la intensidad, desde las aproximantes cerradas hasta las aproximantes vocálicas; o, visto desde otra perspectiva, se puede establecer un continuo según el grado de constricción. 


\section{Referencias bibliográficas}

Boersma, P. y Weenink, D. (1992). Praat: doing phonetics by computer (Versión 5.3.77) [Software] Disponible en: www. praat.org

Borland Delorne, K. (2004). La variación y distribuición alofónica en el habla culta de Santiago de Chile. Onomázein 10 (2), pp. $103-115$.

Croese, R. (1980). Estudio dialectológico del mapuche. Estudios Filológicos 15, pp. 7- 38.

Echeverría, M. (1964). Descripción fonológica del mapuche actual. Boletín del Instituto de Filología de la Universidad de Chile, pp. 13-59.

Figueroa Candia, M. (2012). Propuesta metodológica para el análisis acústico de segmentos aproximantes. (Tesis de maestría no publicada). Pontificia Universidad Católica de Chile, Santiago, Chile.

Henríquez, M. y Salamanca, G. (2012). Rasgos prominentes de la fonología segmental del chedungun hablado por escolares de Alto Bío-Bío. Alpha, 34, pp. 153-171.

Ladefoged, P. (1964). A Phonetic Study of West African Languages. Cambridge: Cambridge University Press.

Lagos, D. (1981). El estrato fónico del mapudungu(n). Nueva Revista del Pacifico, pp. 42-66.

Martínez Celdrán, E. (1998). Análisis espectrográfico de los sonidos del habla. Barcelona: Ariel.

Martínez Celdrán, E. (2013). Caracterización acústica de las aproximantes espirantes en español. Estudios de Fonética Experimental, XXII, pp. 11-35.

Pérez, H. E. (2007) Estudio de la variación estilística de la serie /b-d-g/ en posición intervocálica en el habla de los noticieros de la televisión chilena Estudios de Fonética Experimental XVI, pp. 227-259. 
Piñeros, C.E. (2009). Estructura de los sonidos del español. New Jersey: Pearson Education, Inc.

Sadowsky, S. y Salamanca, G. (2011). El inventario fonético del español de Chile: principios orientadores, inventario provisorio de consonantes y sistema de representación (AFICL). Onomázein 24(2), pp. 61-84.

Sadowsky, S., Painequeo, H., Salamanca, G., Avelino, H. (2013). Mapudungun. Journal of the International Phonetic Association, 43 (1), pp. 87-96

Salamanca, G. (1997). Fonología del pehuenche hablado en Alto Bío-Bío. R.L.A., Revista de Lingüística Teórica y Aplicada, 35, pp.113-124.

Salas, A. (1976). Esbozo fonolófico del mapudungu, lengua de los mapuches o araucanos de Chile central. Estudios Filológicos, 11, pp.143-153.

Simonet, M., J.I. Hualde \& M. Nadeu. (2012). Lenition of /d/ in spontaneous Spanish and Catalan. Proceedings of InterSpeech. The Conference of the International Speech Communication Association (ISCA). Portland, Oregon. (4 p.)

Sola Prado, A. (2011). Las aproximantes $[\beta, ð, \gamma]$ del español en habla espontánea. PHONICA, vol. 7, pp. 118-140. 